\title{
Mechanical and microstructure studies on the modification of CA film by blending with PS
}

\author{
P MEENAKSHI, S E NOORJAHAN, R RAJINI, U VENKATESWARLU, \\ C ROSE and T P SASTRY* \\ Central Leather Research Institute, Adyar, Chennai 600 020, India
}

MS received 24 September 2001; revised 18 October 2001

\begin{abstract}
Dried Musa paradiciaca (banana) stem and veins of the leaves, which were hitherto discarded as a waste, were collected and used as starting material for the preparation of cellulose and cellulose acetate. This cellulose acetate was mixed with polystyrene to form blend of cellulose acetate-polystyrene in order to provide enhanced stability and extended utility to the end products. The films of these composites or their individual partners were made separately and studied for their mechanical properties, chemical modification and morphological changes. We report here that banana stem is good source of cellulose and that cellulose completely undergoes modification upon esterification.
\end{abstract}

Keywords. Musa paradiciaca (banana) plant; cellulose acetate; polystyrene; blends.

\section{Introduction}

Polyesters and polysaccharides represent two traditional and the most useful hydrophobic and hydrophilic polymers, respectively. Polysaccharides such as cellulose, in its various forms, constitute about half of all of the polymers consumed industrially in the world (Li et al 1999). Poly methyl methacrylate (PMMA), polylactic acid, polystyrenes, polyurethanes and an innumerable number of polymers are used for various industrial and biomedical purposes. Composites/blends containing both synthetic and biodegradable polymers will impart novel physical characteristics to the materials which can be used for various purposes like shaped articles, water repellent materials, biomedical equipment etc.

Cellulose acetate (CA) is universally recognized as the most important organic ester of cellulose owing to its extensive applications in fibre, plastics and coatings. Cellulose acetates are prepared by reacting cellulose with acetic anhydride, utilizing acetic acid as a solvent and perchloric acid or sulphuric acid as a catalyst (Tanghe et al 1963). Acetate fibres of cellulose have lower strength and abrasion resistance than most other manmade fibres and are frequently used with nylon or polyester in combination yarns (Platt and Wallace 1983). In recent years, lot of modifications were carried out on cellulose acetate which enabled its use in pharmaceutical, textile, chemical and leather industries. Liu et al (1996) prepared reverse osmosis membrane by graft copolymerizing acrylonitrile onto cellulose acetate. They also

\footnotetext{
*Author for correspondence
}

reported optimum conditions for getting a satisfactory product. Lee et al (1996) graft copolymerized allyl isocyanate (AIC) with partially hydrolyzed cellulose acetate in pyridine to introduce polymerizable vinyl groups through urethane linkage. The reaction was also carried out using different initiators such as $\mathrm{AIBN}, \mathrm{Bz}_{2} \mathrm{O}_{2}$, $\mathrm{K}_{2} \mathrm{~S}_{2} \mathrm{O}_{8}$ and different monomers such as acrylonitrile, acrylic acid and acrylamide.

Whereas styrene polymers have some unique properties which make them useful in wide range of products, polystyrene (PS) has more diverse uses than any other polymer because of its excellent physical properties, ease of fabrication with a variety of equipment and low cost. The principal applications of polystyrene include packaging, appliances for construction, automotive parts, toys, housewares, medicalware, disposable food services and electronic equipment (Platt and Wallace 1983).

Sun et al (1998) prepared semi interpenetrating polymer networks containing cellulose acetate butyrate and poly acrylic ester. These networks had good light fastness and flexibility. Sivakumar et al (1998) used cellulose acetate-polyurethane blends as ultrafiltration membranes for selectively isolating a mixture of proteins. Jumei and Wenwin (1996) prepared a super hygroscopic capsule membrane by encapsulating acrylic acid-starch graft copolymer using cellulose acetate.

In the present study cellulose is prepared from leave veins and stem of banana and the same is converted to cellulose acetate by acetylation reaction. The importance of CA and PS in various industries have long been known individually. But the combined effect of these two polymers in improving biomaterial itself and the functionality of the same is yet to be understood. Moreover, novelty of 
this study lies in the identification of the dried banana plant (a huge agricultural waste in India) for the preparation of CA. In the present study CA-PS blended film was made and its properties as a film/sheet was studied in order to presume its possible role when used for different applications.

\section{Experimental}

\subsection{Materials}

Dried banana stem and central vein of the leaves (BSV) were collected from nearby garden and used as raw material. Used pens made of PS were collected from fellow researchers.

2.1a Preparation of cellulose acetate: Cellulose acetate was prepared by modifying the method described in earlier studies (Tanghe et al 1963). The raw material was cut into $1 \times 1^{\prime \prime}$ pieces and boiled with $1 \mathrm{~N}$ sodium hydroxide solution for $2 \mathrm{~h}$ to remove non-cellulosic materials. Later, it was washed with water to attain $\mathrm{pH} 7 \cdot 0$ and sun dried to get a moisture content of about 11-12\%. Material thus obtained was ground to $100-700 \mu \mathrm{m}$ size powder in a Wiley mill and termed as banana cellulose (BC).

(i) Acetylation of cellulose: To $10 \mathrm{~g}$ of $\mathrm{BC}, 50 \mathrm{ml}$ of acetic acid, $100 \mathrm{ml}$ of toluene and $0.5 \mathrm{ml}$ of perchloric acid $(72 \%)$ were added and stirred vigorously. After one min, $50 \mathrm{ml}$ of acetic anhydride was added and the stirring was continued for $15 \mathrm{~min}$. Later, an equal volume of water was added to the reaction mixture to precipitate CA. The degree of acetylation of cellulose was determined by the method described by Tanghe et al (1963) and found out to be $2 \cdot 83$.

(ii) Preparation of films: A film of CA was made from solution containing CA $(3 \% \mathrm{w} / \mathrm{v})$ in a mixture of dichloromethane and methanol (90:10). Use and throw polystyrene material was dissolved in the above said solvent mixture and the solution (16\% w/v) was used for the preparation of PS film or the film of its composite with $\mathrm{CA}$ with different compositions as shown in table 1 . The

Table 1. Mechanical properties of CA-PS blends.

\begin{tabular}{lcccc}
\hline Sample & $\begin{array}{c}\text { CA solution } \\
(\mathrm{ml})\end{array}$ & $\begin{array}{c}\text { PS solution } \\
(3 \% \mathrm{ml}) \\
(16 \% \mathrm{w} / \mathrm{v})\end{array}$ & $\begin{array}{c}\text { Elongation } \\
\text { at break } \\
(\%)\end{array}$ & $\begin{array}{c}\text { Tensile } \\
\text { strength } \\
\left(\mathrm{kg} / \mathrm{cm}^{2}\right)\end{array}$ \\
\hline 1 & 25 & 75 & $6 \cdot 1$ & $31 \cdot 01$ \\
2 & 50 & 50 & $4 \cdot 8$ & $30 \cdot 12$ \\
3 & 75 & 25 & 4.46 & $7 \cdot 76$ \\
4 & 100 & - & $4 \cdot 11$ & 0.97 \\
5 & - & 100 & $11 \cdot 32$ & 32.46 \\
\hline
\end{tabular}

Thickness of the film in all the samples is $1.00 \mathrm{~mm}$. solution was evaporated in polythene trays at $30^{\circ} \mathrm{C}$ to get $1 \mathrm{~mm}$ thickness films.

2.1b Characterization: The analysis of the products prepared viz. BC, CA film, PS film, CA-PS blend, were carried out using the following methods.

(i) Tensile strength: Two dumbbell shaped specimen of $4 \mathrm{~mm}$ wide and $10 \mathrm{~mm}$ long, were punched out of the film prepared. Mechanical properties such as tensile strength and percentage strain at break were measured using Instron 4501 model tensile testing system at an extension rate of $100 \mathrm{~mm} / \mathrm{min}$, after fixing the samples in the holders.

(ii) Infrared spectroscopy: To provide proof of conversion of $\mathrm{BC}$ into $\mathrm{CA}$ and the chemical nature of the individual constituents in the blend, IR spectra of various experimental samples were taken at a wavelength of $4000-400 \mathrm{~cm}^{-1}$ by using Nicolet Impact 400 Fourier Transform infrared spectrophotometer using $\mathrm{KBr}$ pellet containing 2-6 mg of the sample.

(iii) SEM: Dried samples were coated with solid ions using an ion coater (fisons sputter coater) under the following conditions: $0 \cdot 1$ Torr pressure, $200 \mathrm{~mA}$ current and $70 \mathrm{~s}$ coating time. Surface structure was visualized by scanning electron microscope (SEM model-LEICA stereo scan 440) using a $15 \mathrm{kV}$ accelerating voltage.

\section{Results and discussion}

Polymer blends are used in increasing areas of application because they are cost effective and have potential for design of materials with properties tailored to a specific use (Chuck and An-Chang 1999). However, the biodegradability of a product is of utmost importance in the present day world to reduce the disposal problem. Therefore, an attempt has been made in this study to combine a biodegradable material like $\mathrm{CA}$, and a use and throw polymeric material so that the resultant product is bio-

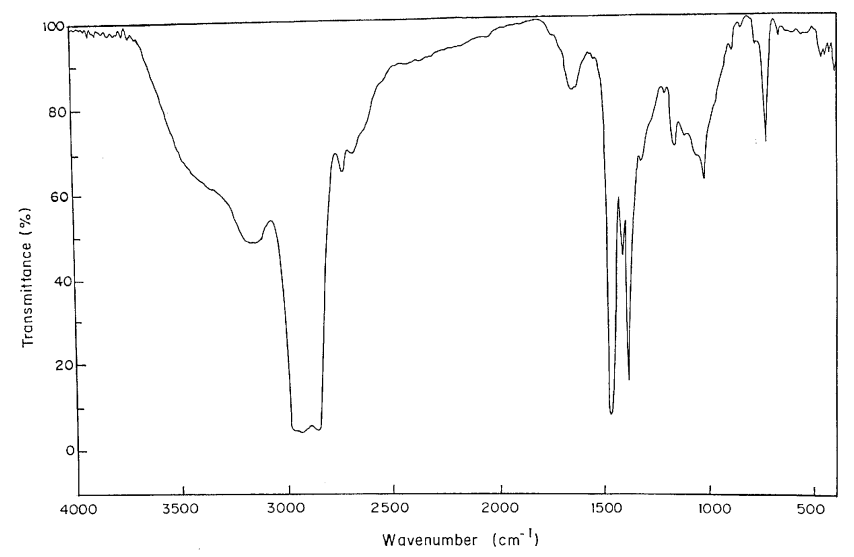

Figure 1. Infrared spectrum of Banana cellulose (BC). 
degradable to some extent and the polymer is reused in a different form to any desired purpose.

\subsection{Tensile strength}

The mechanical properties of various compositions of CA and PS blend are presented in table 1. Films of CA and PS were also made separately as controls. As expected, PS film has shown comparatively better tensile strength $\left(32.45 \mathrm{~kg} / \mathrm{cm}^{2}\right)$ and elongation at break $(11.32 \%)$ because of its polymeric chain nature. On the contrary, CA, a substituted linear polysaccharide chain containing $1 \rightarrow 4$, $\beta$-glycoside linkages exhibited poor mechanical properties. The poor mechanical properties exhibited by CA may be attributed mainly due to its porous nature (figure 6). It has been reported that most of the man-made fibres like nylon, polyester, etc have more strength than the fibres or films made out of biopolymers (Platt and Wallace 1983).

Therefore, a blend containing varied compositions of substituted biopolymer and PS were prepared with a view to (i) utilize the natural material and (ii) impart strength and stability, respectively. According to the results obtained (table 1) in the present study, the tensile strength of the blend film containing $50 \mathrm{ml} \mathrm{CA}$ solution $+50 \mathrm{ml}$ PS solution was almost comparable with that of film made using PS alone. However, $100 \%$ elongation could not be achieved in the blend for the known reason. This is clearly seen in table 1 that with the increase in CA concentration, the elongation property is gradually decreased. Whereas, there is a sudden decrease in the tensile strength after further increase in the $\mathrm{CA}$ concentration. The interpore distance in the CA (figure 6) is much less (i.e. it is more porous) than observed in the PS (figure 7). This attributes better mechanical properties to PS than CA. Hence when the amount of CA is increased in the CA-PS blend, there is a down trend in the mechanical properties.

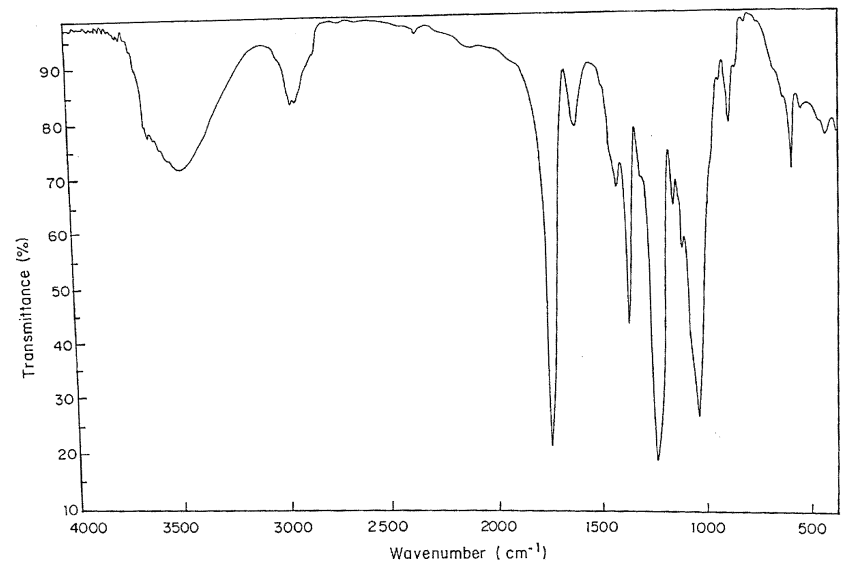

Figure 2. Infrared spectrum of cellulose acetate (CA).

\subsection{IR spectroscopy}

The infrared spectra taken for BC, CA, PS and CA-PS blend are shown in figures $1-4$, respectively. The IR spectrum of BC of this study is similar to the one reported earlier (Brugal 1962). The characteristic - $\mathrm{OH}$ stretching vibration with strong intensity broad band around 3700 $3100 \mathrm{~cm}^{-1}$ with a broad peak from $3300-3100 \mathrm{~cm}^{-1}$ is seen. The $-\mathrm{OH}$ inplane deformation vibration from 1460 $1324 \mathrm{~cm}^{-1}$ and $\mathrm{C}-\mathrm{O}$ stretching vibration bands from $1160-1025 \mathrm{~cm}^{-1}$ are also seen. The pattern of bands confirms the nature of the cellulose.

Whereas in the IR spectrum of CA (figure 2) the $-\mathrm{OH}$ peak obtained for cellulose in the figure is almost suppressed. Instead, a predominant ester carbonyl band with a strong intensity is seen at $1755 \mathrm{~cm}^{-1}$. This must be representing the acetylate form of cellulose wherein the $-\mathrm{OH}$ groups are converted into acetate group through ester linkage.

The IR spectrum of PS is comparable with that of earlier reports (Brugel 1962; Simons 1978). A C-C stretching vibration band is seen at $1601 \mathrm{~cm}^{-1}$. The bands

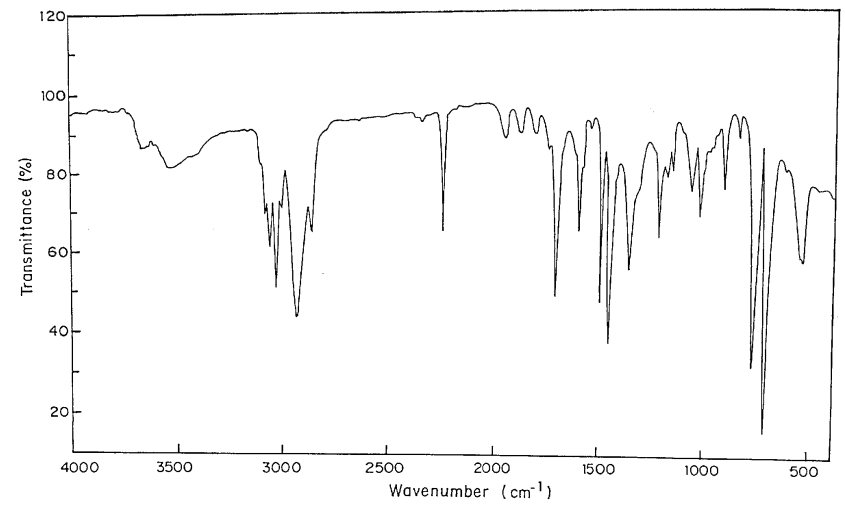

Figure 3. Infrared spectrum of polystyrene (PS).

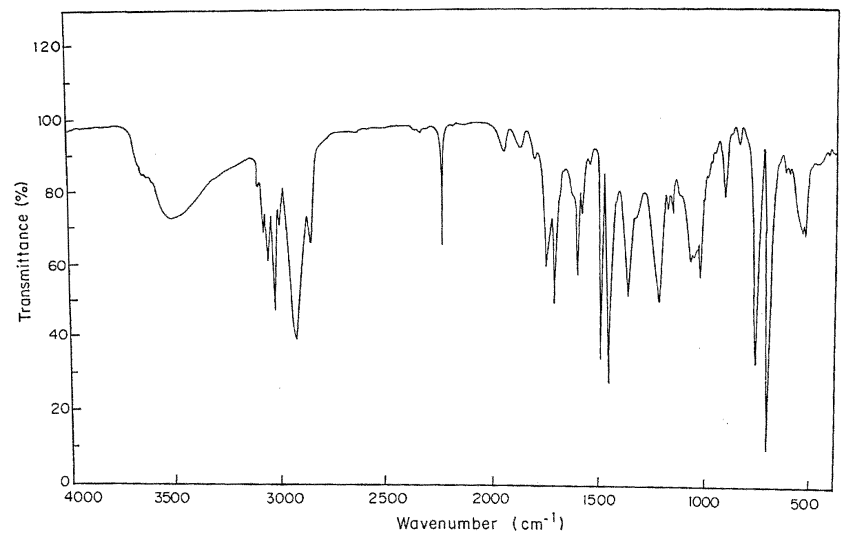

Figure 4. Infrared spectrum of CA-PS blend. 
at $1493 \mathrm{~cm}^{-1}$ and $1450 \mathrm{~cm}^{-1}$ represents alkyl substitution. The $\mathrm{C}-\mathrm{H}$ deformation vibration band of benzene ring hydrogens (5 adjacent hydrogens) is observed at $761 \mathrm{~cm}^{-1}$. Ring deformation vibration is observed at $701 \mathrm{~cm}^{-1}$. C-H stretching vibrations of ring hydrogens are seen from
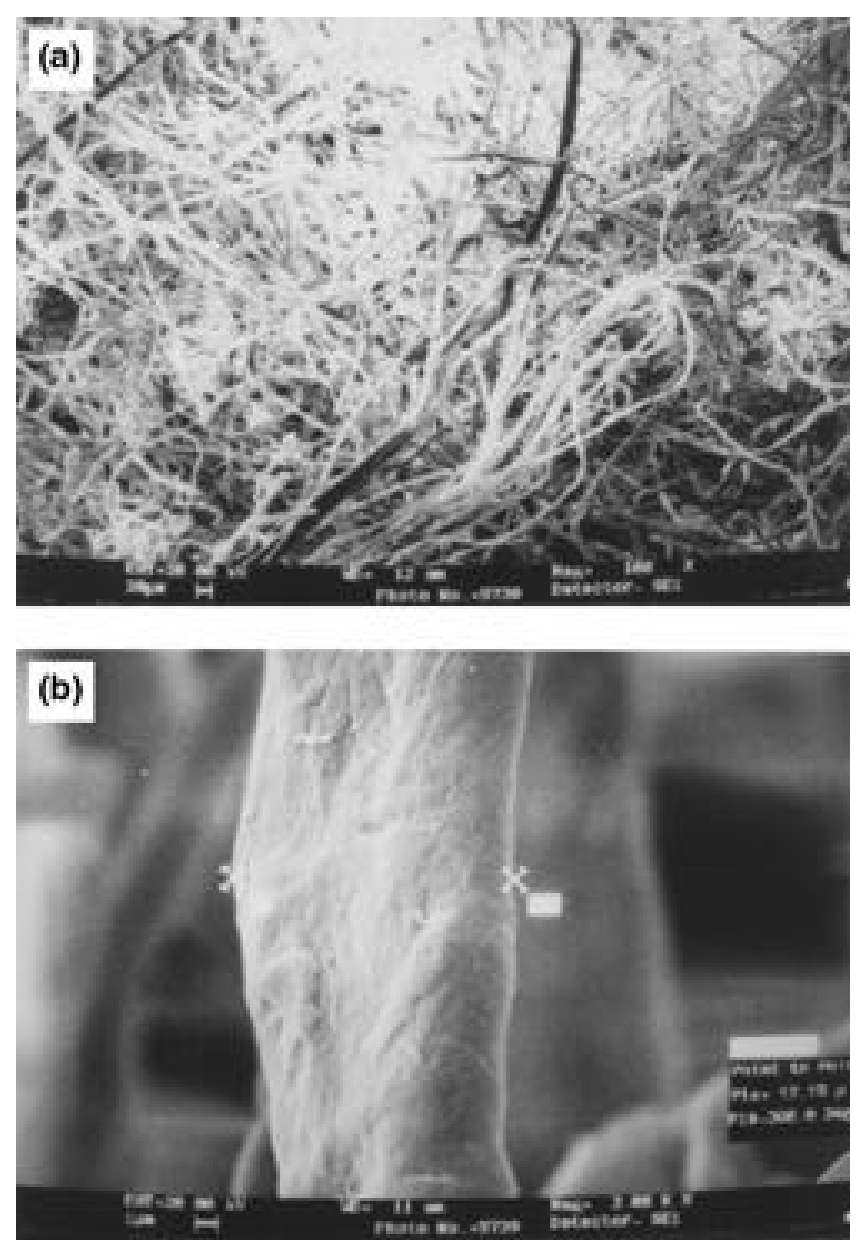

Figure 5. Scanning electron micrographs of $\mathrm{BC}(\mathbf{a})(\times 100)$ and (b) $(\times 3000)$.

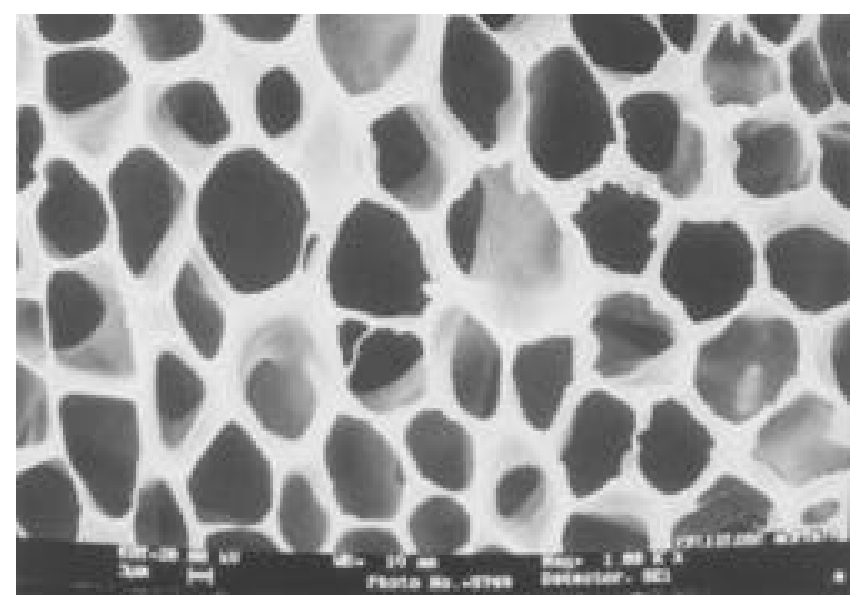

Figure 6. Scanning electron micrograph of CA $(\times 1000)$.
$2926-3100 \mathrm{~cm}^{-1}$ (5 bands). This confirms IR spectral nature of PS.

CA-PS blend gave the characteristic peaks of both CA and PS. As there are no shifts of the peaks of any group in CA-PS spectrum, it is confirmed that CA-PS remained as physical blend and no chemical reaction had taken place between the two compounds.

\subsection{Scanning electron microscopy}

The beautiful fibrous structure of $\mathrm{BC}$ is seen in figure $5 \mathrm{~A}$. In figure $5 \mathrm{~B}$, surface morphology of a single fibre is seen. The surface is smooth even though it is not uniform. The SEM picture of CA (figure 6) shows a wonderful mosaic like structure with uniform porosity. The regular arrangement of pores in CA film reminds the structure of beehive. The structure of PS film (figure 7) is also porous like CA, but the regularity in the size and shape of the pore is lacking. The pore size of CA-PS film (figure 8) looks

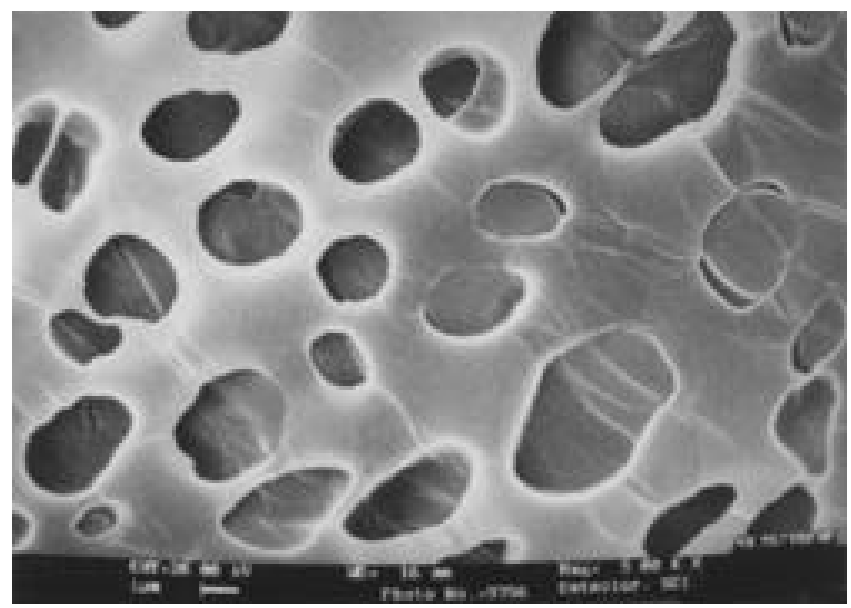

Figure 7. Scanning electron micrograph of PS $(\times 5000)$.

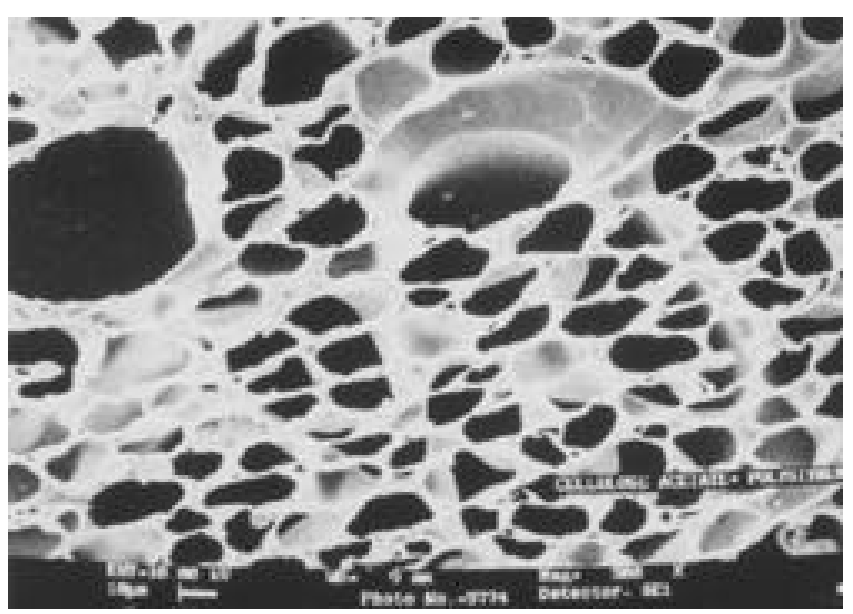

Figure 8. Scanning electron micrograph of CA-PS blend $(\times 500)$. 
regular, which may be due to the presence of $\mathrm{CA}$ that dominates the morphological structure of blend $50 \mathrm{ml}$ of CA solution $+50 \mathrm{ml}$ of PS solution.

\section{Conclusions}

Both CA and PS were found to be soluble in the solvent mixture of dichloromethane-methanol. The resultant clear solution was able to form a smooth translucent film with adequate handling strength. The CA owing to its inertness to biological system could be used in biomedical applications. However, due to its poor mechanical property exhibited here, the idea of blending with PS was mooted in this investigation. This synthetic polymer apart from its compatible nature imparts strength to the film.

Effective utilization of the modified cellulose and the waste PS materials would help in solving the disposable problem. Additionally, the end product, in the form of filtration membrane with variety of industrial and medical application, would fetch value addition.

\section{References}

Brugal W 1962 in An introduction to infrared spectroscopy (Great Britain: Methuen \& Co) p. 352

Chuck Y and An-Chang S 1999 Macromolecules 323637

Jumei W and Wenwin W 1996 Xian Weisu Kexue; Yu Zishu 436

Lee S, Kim J W, Oh Y S and Lee H S 1996 Pollimo 201014

Li J, Xie W, Cheng H N, Nickol R G and Wang P G 1999 Macromolecules 322789

Liu Y, Chen D, Wang L, Chen Y, Hong Y and Gong M 1996 Shuichuli Jishu 22323

Platt A E and Wallace T C 1983 in Encyclopedia of chemical technology (New York, Toronto: John Wiley \& Sons) 21 p. 801

Simons William W 1978 in Sadtler Handbook of infrared spectra (Pennsylvania, USA: Sadtler Research Laboratories)

Sivakumar M, Mohan D and Rangarajan R 1998 Polym. Int. 47 311

Sun J, Litu Z and Wie D 1998 Pige Huagong 1514

Tanghe L J, Genung L B and Mench J W 1963 in Advances in carbohydrate chemistry (New York, London: Academic Press) 3 p. 193 